\title{
$N$-Acetylcysteine reduces respiratory burst but augments neutrophil phagocytosis in intensive care unit patients
}

\author{
Axel R. Heller, MD; Gesine Groth, MD; Susanne C. Heller, MD; Raoul Breitkreutz, MD; Thomas Nebe, MD; \\ Michael Quintel, MD, PhD; Thea Koch, MD, PhD
}

Objective: The antioxidant $\mathrm{N}$-acetylcysteine (NAC) has been shown to attenuate septic tissue injury. To evaluate whether NAC affects host defense mechanisms in critically ill patients, thus predisposing to increased risk of infection, the current study focuses on neutrophil phagocytotic and burst activity after treatment with NAC.

Design: Prospective, randomized, clinical trial.

Setting: Twelve-bed operative intensive care unit in a university hospital.

Patients: Thirty patients diagnosed with sepsis/systemic inflammatory response syndrome, or multiple trauma.

Interventions: Patients were randomly assigned to receive either NAC $(n=15)$ for 4 days in increasing dosages (day 1: $6 \mathrm{~g}$; day 2: $12 \mathrm{~g}$; days 3 and 4: $18 \mathrm{~g}$ ) or a mucolytic basis dosage of NAC $(3 \times 300 \mathrm{mg} /$ day [control]; $n=15)$, respectively.

Measurements and Main Results: Blood samples were taken before NAC high-dose infusion (day 1), after increasing doses of NAC (days 3 and 5) and 4 days after the last high-dose treatment (day 8). Neutrophil oxidative burst activity after stimulation with Escherichia coli and polymorphonuclear phagocytosis were de- termined in a flow cytometric assay. Baseline values of polymorphonuclear functions were comparable in both groups. NAC highdose treatment resulted in a significantly improved phagocytosis activity compared with control patients. In contrast to this, polymorphonuclear burst activity was significantly reduced in the NAC high-dose treated group on day 3.

Conclusion: These findings suggest that infusion of NAC in high doses affects granulocyte functions in critically ill patients. Antimicrobial host defense requires the effective sequence of cell adhesion, phagocytosis, and bactericidal respiratory burst. The enhanced phagocytotic activity might be a compensatory mechanism in states of impaired respiratory burst to maintain tissue sterility. For certain mechanisms of disease, the effects observed might be favorable (e.g., ischemia/reperfusion, endothelial cell activation), for others (infection) this might be detrimental. (Crit Care Med 2001; 29:272-276)

KeY WoRDS: $\mathrm{N}$-acetylcysteine; antioxidants; neutrophils; respiratory burst; phagocytosis; flow cytometry; sepsis; mucolysis; tissue injury; host defense
A variety of experimental studies provide evidence that tissue injury associated with severe sepsis and multiorgan failure is mediated in part by leukocyte activation and subsequent release of reactive oxygen intermediates and proteinases into the microvasculature (1). Free oxygen radi-

From the Department of Anesthesiology and Intensive Care Medicine, University Hospital Carl Gustav Carus, Dresden, Germany (Drs. A. Heller, S. Heller, and Koch), the Department of Anesthesiology and Intensive Care Medicine (Ms. Groth and Dr. Quintel) and the Institute of Clinical Chemistry (Dr. Nebe), University Hospital Mannheim, Germany, and the Department of Immunochemistry, Deutsches Krebsforschungszentrum (DKFZ), Heidelberg, Germany (Dr. Breitkreutz).

Supported, in part, by the Research Fund of the Faculty of Clinical Medicine Mannheim, University of Heidelberg.

Address requests for reprints to: Axel R. Heller, MD, Klinik fur Anaesthesiologie und Intensivtherapie, Universitatsklinikum Carl Gustav Carus, Fetscherstrasse 74, D-01307 Dresden, Germany. E-mail: heller-a@rcs.urz.tu-dresden.de cals are able to attack polyunsaturated fatty acid chains of membrane lipids, thus initiating a peroxidation process (2). Peroxidation destroys the functional integrity of the cell membrane, culminating in an acute increase of capillary permeability. It has been shown that leukocyte depletion or inhibition of neutrophil adhesion and oxygen radical generation reduce tissue injury and confer a survival advantage in various animal models of shock, sepsis $(3,4)$, and ischemial reperfusion injury $(5,6)$. In the healthy organism, a protective system of endogen antioxidants counteracts radical formation. Enzymatic and nonenzymatic systems exist to prevent cell and tissue injury. The main enzymatic system is the glutathione peroxidase system that detoxifies both organic and anorganic peroxides. An overwhelming release of peroxides, however, overcomes the antioxidative capacity and induces oxidative stress. Recently Metnitz and coworkers described reduced levels of antioxidative capacity in patients suffering from acute respiratory distress syndrome (7).
Thus, in order to attenuate oxygen radical mediated organ damage, adjuvant therapy with various antioxidants has been investigated in animal studies $(8,9)$ and clinical trials $(10,11)$. The supplementation of various antioxidants, especially of $\mathrm{N}$-acetylcysteine (NAC), has been shown to attenuate acute lung injury as assessed by reduced lung permeability, which seems to be related to free radical scavenging and inhibition of the neutrophil oxidative burst (8). In few clinical trials, NAC shortened the duration of acute lung injury $(10,11)$ whereas other studies provided conflicting results $(12,13)$.

NAC represents a safe agent with a wide toxic-therapeutic window. Increasing numbers of animal studies indicate efficacy of NAC in the prevention and therapy of lung injury involving toxic oxygen species (14). NAC, a thiol compound, is a radical scavenger, working either directly or by means of the thiol group or as a precursor of glutathione (2). In addition to the radical scavenger effect of NAC, it may counteract neutro- 
phil activation and aggregation in the lung (15).

In the last years, evidence has accumulated that defects in polymorphonuclear functions such as impaired chemotaxis, adherence, phagocytosis, and bacterial killing may cause an increased incidence of sepsis and multiple organ failure (16). Because one important mechanism by which phagocytes destroy microbial organisms is the production of reactive oxygen intermediates (17), one might hypothesize that NAC impairs host immune response by interfering with the leukocytal production of essential toxic oxygen species (18). To elucidate this controversy of beneficial and adverse effects (19), the current study was designed to investigate whether NAC affects neutrophil phagocytosis and respiratory burst in critically ill patients, thus predisposing the host to increased risk of bacterial infections.

\section{MATERIALS AND METHODS}

Patients. With institutional review board approval and written informed consent from the patients or their relatives, we prospectively enrolled 30 patients treated for septic shock in the 12-bed Intensive Care Unit (ICU) of the Department of Anesthesiology and Operative Intensive Care Medicine, University Hospital of Mannheim, from February to December 1997. All patients were mechanically ventilated and had clinical and laboratory variables that fulfilled the criteria of septic shock as outlined in the 1992 consensus conference (20). After enrollment in this study, the patients were randomly assigned to either the NAC high-dose or the NAC low-dose group. Inclusion of patients to the respective groups was achieved by computer-derived block randomization. Solutions were prepared in the central pharmacy of the university hospital, delivered to the ICU, and further handled by a nurse who was unaware to the study protocol. The investigators were blinded to the infused drug. The first day that patients fulfilled the criteria for septic shock after admission to the ICU was defined as day 1 . Patients were excluded from the study if they were known to suffer from neoplastic disease, had an acquired immunodeficiency syndrome, or were pregnant.

Interventions. Patients of the control group received $300 \mathrm{mg}$ of NAC three times a day intravenously for mucolysis, at least until day 8 . The 15 patients in the treatment group continuously received NAC intravenously for 4 days in increasing doses by means of an infusion pump (Volumed $\mu$ VP5000; Fresenius, Bad Homburg, Germany) in $240 \mathrm{~mL}$ sterile saline solution $0.9 \%$ (infusion rate: $10 \mathrm{~mL} / \mathrm{h}$ ). Within the first $24 \mathrm{hrs}, 6 \mathrm{~g}$ of NAC were infused. Then the dosage was increased to $12 \mathrm{~g} /$ day (day 2) and to $18 \mathrm{~g} /$ day (days 3 and 4). On day 5, NAC high-dose treatment was ceased and all patients received mucolytic doses of $300 \mathrm{mg}$ NAC three times a day, at least until day 8 .

Blood Samples. In addition to the routine laboratory measurements, including peripheral blood cell counts and biochemical analysis, $2 \mathrm{~mL}$ of whole blood were obtained for days 1 (before NAC- treatment), 3, 5, and 8 . The collected central venous blood was heparinized with $10 \mathrm{U} / \mathrm{mL}$ (Liquemin 25000 , Roche, Grenzach-Whylen, Germany).

Polymorphonuclear Burst Activity. The amount of intracellular oxygen radical production was determined in freshly drawn heparinized whole blood. A test kit (Bursttest, Orpegen Pharma, Heidelberg, Germany) for the determination of the oxidative burst of leukocytes in whole blood was used. Singlecell analysis was performed by flow cytometry. The method of the quantitative assay for monitoring the oxidative burst was described in detail previously (21). Briefly, two assays (100 $\mu \mathrm{L}$ ) of each sample were incubated (10 mins; $37^{\circ} \mathrm{C}$ ) with either phosphate-buffered saline (negative control), or with Escherichia coli that had been opsonized with antibodies and complement from pooled sera. E. coli stimulates production of reactive oxygen intermediates. After the oxidation step (incubation with substrate 10 mins; $37^{\circ} \mathrm{C}$ ) in which the nonfluorescent substrate, dihydrorhodamine 123 was taken up by the phagocytes and converted during the respiratory burst to a green fluorescent compound (rhodamine 123), the whole blood was lysed and fixed. This oxidation is highly specific for the respiratory burst activity. To guarantee that no cell debris or dead cells or bacteria interfere with the measurement, DNA staining was performed with propidiumiodide (red fluorescence, FL-3). 15,000 cells of each sample were measured with a laser flow cytometer (FACScan/Lysis II, Becton Dickinson, Heidelberg, Germany) using blue-green excitation light (488 nm argon laser). Photomultiplier gain setting was adjusted to register all intensities of cellular fluorescence within the scale of 256-channel resolution (four decades). Neutrophils were identified by their characteristic size to granularity ratio. Only the selected cell population was considered for further analysis (multiple document interface for Windows 3.1 [WinMDI 2.0] by J. Trotter, Scripps Research Institute, La Jolla, CA). During data acquisition, a "live" gate was set in the red fluorescence dot plot on those events that had at least the same DNA content as human diploid cells. Only these cells were examined. For characterization of the fluorescence distribution, the mean values (FL-1) were used, which indicate the amount of rhodamine 123 per cell.

PMN Phagocytosis Activity. A test kit (Phagotest, Orpegen Pharma, Heidelberg, Germany) for the determination of the phagocytic activity of leukocytes in whole blood was used. Single-cell analysis was performed by flow cytometry. The method of the quantitative assay for investigating the phagocytic activity was described in detail previously (22). Two assays of each sample $(2 \times 100 \mu \mathrm{L})$ were incubated with fluorescein isothiocyanate (FITC)-marked opsonized E. coli, either at $0^{\circ} \mathrm{C}$ (control) or at $37^{\circ} \mathrm{C}$ for 10 mins. Cells of the phagocytic system have receptors for a complement component $(\mathrm{C} 3 \mathrm{~b})$ and for the constant part of the immunoglobulin molecule $(\mathrm{Fc})$ mediating the adhesion of the bacteria to the cell surface and subsequent engulfment. During the incubation period the green fluorescent (FITC) marked $E$. coli were ingested in the $37^{\circ} \mathrm{C}$ assay. To exclude extracellular bacteria from measurement, they were quenched with a staining solution. The further steps such as lysis, fixation, DNA-staining, and data acquisition by flow cytometer were performed in the same manner as in the burst assay. The phagocytosis activity of leukocytes was determined by the content of FITC-marked $E$. coli in the phagocytic cells, expressed as mean channel fluorescence (FL-1) per cell.

Statistics. Data are presented as arithmetic mean \pm SEM. Results of phagocytosis and oxidative burst activity are expressed as mean channel fluorescence per cell. Differences among both groups were tested with Student's $t$-test or chi-square test. Within-group analysis was performed with one-way analysis of variance (ANOVA) followed by StudentNewman-Keuls multiple comparison procedure. Significance was accepted at $p<.05$. Analysis was performed using SPSS for MS Windows (Release 9.0.1, SPSS, Chicago, IL).

\section{RESULTS}

Clinical Characteristics of the $\mathrm{Pa}$ tients. Demographic characteristics of the patients concerning age, gender, ICU stay, Acute Physiology and Chronic Health Evaluation (APACHE) II score and predicted risk of hospital death (23) at entry, survival, and diagnoses are summarized in Table 1. There were no significant differences between both groups in basis data at entry. Patients in both groups required inotropic support, mechanical ventilation, and intensive care for similar length of time. Mortality was higher in the NAC high-dose group, but the difference did not reach statistical significance.

Neutrophil Function. Baseline values of polymorphonuclear functions before intervention did not differ significantly among the two groups (Fig. 1). NAC high-dose treatment resulted in a significantly improved phagocytosis activity after increasing the dosage to 12 and $18 \mathrm{~g}$ per day (3.2-fold day $3, p<.05 ; 6.1$-fold day $5, p<.01)$ as compared with NAC 


\begin{tabular}{lccr}
\hline \multicolumn{1}{c}{ Patients } & $\begin{array}{c}\text { NAC High Dose } \\
(\mathrm{n}=15)\end{array}$ & $\begin{array}{c}\text { NAC Low Dose } \\
\text { (Control) } \\
(\mathrm{n}=15)\end{array}$ & $p$ \\
\hline Age, yrs & $42.5 \pm 5.0$ & $48.5 \pm 4.9$ & .40 \\
Gender, male/female & $11 / 4$ & $11 / 4$ & 1.00 \\
ICU stay, days & $27.2 \pm 4.2$ & $21.3 \pm 2.8$ & .25 \\
APACHE II score at entry & $25.1 \pm 4.6$ & $21.8 \pm 4.2$ & .60 \\
Risk of hospital death, \% & $51.8 \pm 5.9$ & $46.0 \pm 5.2$ & .46 \\
Nonsurvivors (\%) & $7(46.7)$ & $4(26.7)$ & .21 \\
Diagnoses & & 4 & 5 \\
Peritonitis & 3 & 1 & 4 \\
ARDS & 3 & 4 & \\
Multiple trauma & 4 & 1 & \\
Pneumonia & 4 & NA & \\
Head injury & 3 & 3 & \\
Pancreatitis & NA & 1 & \\
Ruptured aortic aneurysm & & & \\
Osteomyelitis & & & \\
\hline
\end{tabular}

NAC, $N$-acetylcysteine; ICU, intensive care unit; APACHE, Acute Physiology and Chronic Health Evaluation; ARDS, acute respiratory distress syndrome; NA, not applicable.

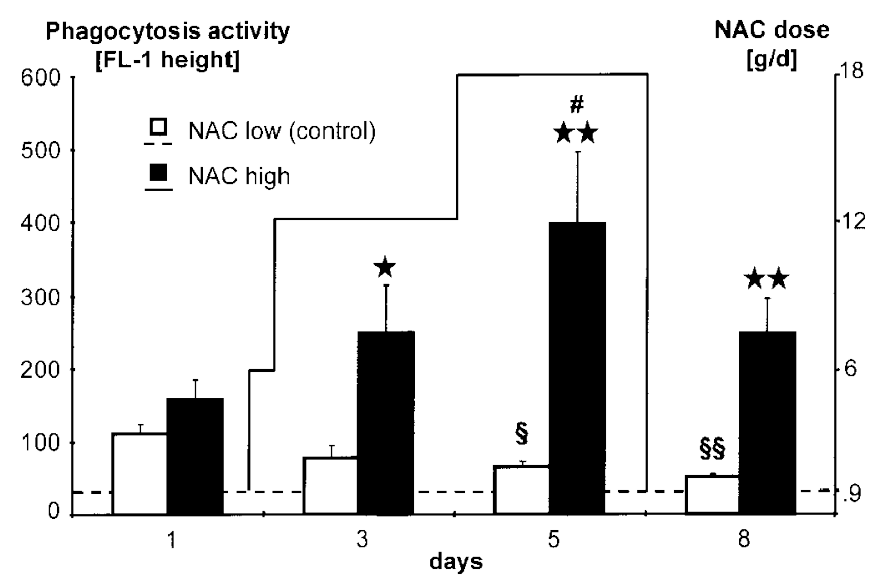

Figure 1. Neutrophil phagocytosis activity (bars) of patients who received $N$-acetylcysteine ( $N A C$ high, $\mathrm{n}=15$ ) in increasing doses (solid line) up to $18 \mathrm{~g} /$ day (day 4) and of control patients (NAC low, 0.9 $\mathrm{g} /$ day, $\mathrm{n}=15)$, respectively. Results are presented as mean channel fluorescence $(F L)$ per cell. Mean values \pm SEM and significant differences between and within the groups are indicated. $(\star \star p<.01 \mathrm{vs}$. $\mathrm{NAC}$ low (control); $\star p<.05$ vs. NAC low (control); $\S \S p<.01$ vs. control day $1 ; \S p<.05$ vs. control day $1 ; \# p<.05$ vs. NAC high day 1$)$.

low-dose patients. Moreover, phagocytosis activity significantly increased 2.5 -fold within the treatment group (day 1 vs. day $5 ; p<.05)$ whereas phagocytosis activity in the NAC low-dose group significantly decreased to $59 \%$ of baseline at day 5 ( $p$ $<.05$ vs. day 1$)$ and to $45 \%$ at day $8(p<$ .01 vs. day 1$)$, respectively. In contrast to this, polymorphonuclear burst activity (Fig. 2) was significantly reduced to $39 \%$ of control levels in the NAC high-dose treated group (day 3; $p<.05$ ) but regained control levels after discontinuation of the NAC high-dose treatment (day $8 ; p<.05$ vs. days 1,3 , and 5 ).

The observed changes in polymorphonuclear function were not associated with significant changes in infection rates or need for antibiotic medication.

\section{DISCUSSION}

Leukocyte activation, subsequent phagocytosis, and release of oxygen radicals play a crucial role in host defense (24). Thus, failure to eliminate inoculated exogenous or translocated endogenous bacteria due to host immunosuppression (25) contributes to the persistence of bacteria in the blood and to the development of septic complications.

The results of the present study provide evidence that NAC high-dose treatment significantly $(p<.05)$ increases neutrophil phagocytosis (Fig. 1) as com-

ogether with previ-
ous findings from
an animal model
in which N-acetylcysteine
(NAC) impaired polymor-
phonuclear burst and bacte-
rial elimination from the
blood, we conclude that NAC
treatment may affect host
defenses in terms of compro-
mised bacterial killing in
critically ill patients.

pared with low-dose NAC administration (control group). Moreover, phagocytosis levels significantly $(p<.05)$ dropped during the observation period in the control group. NAC, however, significantly raised neutrophil phagocytosis from day 1 to day 5. In contrast, neutrophil respiratory burst was significantly suppressed under NAC treatment on day 3 (Fig. 2), but levels significantly increased after termination of high-dose NAC therapy, reaching almost control values at day 8 . In the NAC low-dose group, spontaneous increase of respiratory burst occurred that might represent a cellular up-regulation of respiratory burst in response to the administration of low-dose NAC in order to maintain oxygen radical-based host defense. The NAC dosage utilized in the current study was similar to concentrations used in various human and animal studies investigating the effect of NAC on septic tissue injury (5). With regards to the reported elimination half-life time (26), increasing doses up to $18 \mathrm{~g} /$ day were continuously administered intravenously to ensure effective concentrations of NAC in the circulation during the observation period. The applied NAC doses did not alter hemodynamic or respiratory parameters in our patients, thus effects of regional perfusion inhomogeneities on neutrophil functions can be excluded. The present data of suppressed respiratory burst after NAC treatment are concordant with data from other investigators $(27,28)$ and a previous animal study 


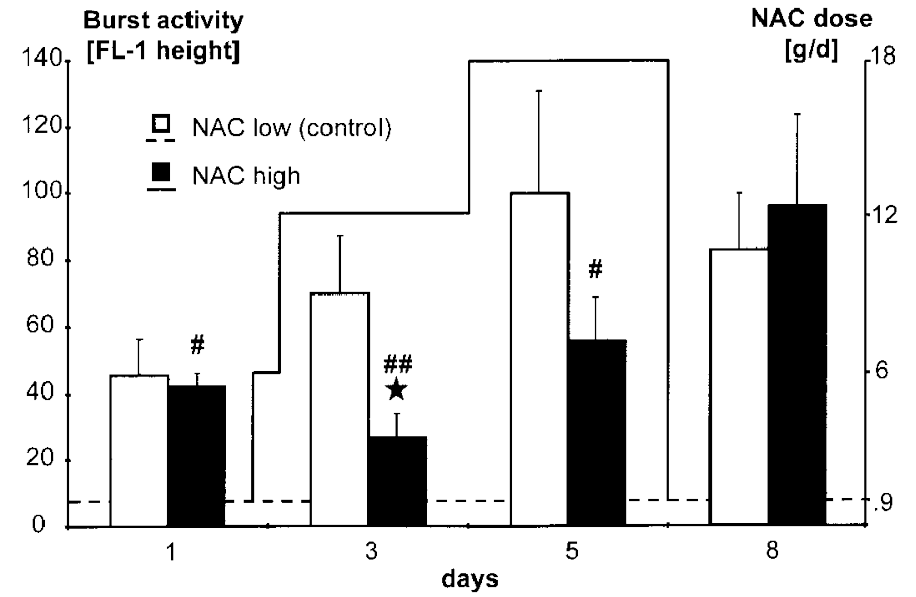

Figure 2. Neutrophil respiratory burst activity (bars) of patients who received $N$-acetylcysteine (NAC high, $\mathrm{n}=15$ ) in increasing doses (solid line) up to $18 \mathrm{~g} /$ day (day 4 ) and of control patients (NAC low, $0.9 \mathrm{~g} /$ day, $\mathrm{n}=15)$, respectively. Results are presented as mean channel fluorescence $(F L)$ per cell. Mean values \pm SEM and significant differences between and within the groups are indicated. $(\star p<.05$ vs. control; \#\#p <.01 vs. NAC high day 8 ; \#p $<.05$ vs. NAC high day 8).

of our group (18). In our rabbit model, reduced respiratory burst activity was accompanied with significantly impaired clearance of exogenously administered bacteria from the blood and significantly increased bacterial colonization in liver, lung, and kidney (18). Moreover, a variety of studies point toward inhibitory effects of NAC on human neutrophil and monocyte chemotaxis and oxidative metabolism (29), T-cell responses including NFkappaB (30), VCAM-1, and E-selectin (31) suppression. NAC effects on VCAM-1 and E-selectin were explained by differences in the redox sensitivity of the NF-kappaB binding to its consensus sequence in the VCAM-1 and E-selectin gene, allowing differential gene expression regulated by the same transcription factor (32).

Conversely to the effects on respiratory burst during NAC administration, polymorphonuclear phagocytosis was significantly enhanced. Accordingly, Linden and co-workers found improved phagocytosis capacity after NAC treatment in human alveolar macrophages (33). In their study Ohman and colleagues provide an explanation for the observation of depressed respiratory burst and augmented phagocytosis. They conclude that the scavenger effect of NAC not only reduces the accumulation of oxidative metabolites per se, but also enhance receptormediated phagocytosis by protecting $\mathrm{Fc}$ (immunoglobulin $\mathrm{G}$ ) receptors from oxidative damage mediated by myeloperoxidase and $\mathrm{H}_{2} \mathrm{O}_{2}$ (34). Antimicrobial host defense requires the effective sequence of chemotaxis, cell adhesion, phagocytosis, and bactericidal respiratory burst.
Whether or not enhanced phagocytosis activity as observed in our study is due to Fc receptor protection or due to an upregulated compensatory mechanism to preserve host defense in states of impaired respiratory burst cannot be answered from the current data. Moreover, the observed dose-dependent effects of NAC (0.9 g, 6 g, 12 g, 18 g, 0.9 g per day) might theoretically be confounded by changes over time. Taking into consideration the short NAC half-life time (26), dose-independent and time-dependent changes appear unlikely. The critical question, however, of whether compromised oxidative burst activity induced by NAC or other antioxidants predisposes the host to an increased susceptibility to infection remains unclear. Until now, studies investigating the relationship between polymorphonuclear function and the development of infections revealed conflicting results. While Duignan et al. (35) and Buffone et al. (36) reported that reduced chemotaxis observed after surgery correlates with the development of postoperative infection and sepsis, other investigators $(37,38)$ did not find any correlation between postoperative neutrophil dysfunction regarding enzyme contents (39) and respiratory burst (40), and the incidence of bacterial infections.

Summarizing our results, NAC was shown to suppress neutrophil oxidative burst activity on the one hand and to augment phagocytosis on the other hand. Together with previous findings from an animal model in which NAC impaired polymorphonuclear burst and bacterial elimination from the blood, we conclude that NAC treatment may affect host defenses in terms of compromised bacterial killing in critically ill patients. For certain pathophysiologic mechanisms, such as ischemia/reperfusion, or endothelial cell activation, the observed effects might be favorable. During infection or septic states, however, reduced burst activity might be detrimental.

\section{ACKNOWLEDGMENTS}

We thank the staff of the Mannheim University Hospital ICU for their kind and generous support, and Kerstin Salomon, Jutta Schulte, Angelika Tapper, Monika Lehmer (Institute of Anesthesiology), and Jutta Christophel (Center of Medical Research, University Hospital of Mannheim, Germany) for excellent technical assistance.

\section{REFERENCES}

1. Tanaka H, Sugimoto H, Yoshioka T, et al: Role of granulocyte elastase in tissue injury in patients with septic shock complicated by multiple organ failure. Arch Surg 1991; 213: 81-85

2. Heffner JE, Repine JE: Pulmonary strategies of antioxidant defence. Am Rev Respir Dis 1989; 140:531-554

3. Walsh CJ, Carey PD, Cook DJ, et al: AntiCD18 antibody attenuates neutropenia and alveolar capillary-membrane injury during Gram-negative sepsis. Surgery 1991; 110: 205-12

4. Lundblad R, Ekstrom P, Giercksky K-E: Pentoxifylline improves survival and reduces tumor necrosis factor, interleukin-6, and endothelin-1 in fulminant intra-abdominal sepsis in rats. Shock 1995; 3:210-215

5. Suzuki M, Inauen W, Kvietys PR, et al: Superoxide mediates reperfusion-induced leukocyte-endothelial cell interactions. $\mathrm{Am} \mathrm{J}$ Physiol 1989; 257:H1740-H1745

6. Hernandez LA, Grisham MB, Twohig B, et al: Role of neutrophils in ischemia-reperfusioninduced microvascular injury. Am J Physiol 1987; 253:H699-H703

7. Metnitz PGH, Bartens C, Fischer M, et al: Antioxidant status of patients with acute respiratory distress syndrome. Intensive Care Med 1999; 25:180-185

8. Davreux CJ, Soric I, Nathens AB, et al: $N$ Acetyl cysteine attenuates acute lung injury in the rat. Shock 1997; 8:432-438

9. Heller S, Weber K, Heller A, et al: Pentoxifylline improves bacterial-clearance in hemorrhage and endotoxemia. Crit Care Med 1999; 27:756-763

10. Bernard GR, Wheeler AP, Arons MM, et al: A trial of antioxidants $N$-acetylcysteine an procysteine in ARDS. The Antioxidant in ARDS Study Group. Chest 1997; 112:164-172

11. Suter PM, Domenighetti G, Schaller M-D, et 
al: $N$-Acetylcysteine enhances recovery from acute lung injury in man: A randomized double blind, placebo-controlled clinical study. Chest 1994; 105:190-194

12. Laurent T, Markert M, Feihl F, et al: Oxidantantioxidant balance in granulocytes during ARDS: Effect of $N$-acetylcysteine. Chest 1996; 109:163-166

13. Jepsen S, Herlevsen $\mathrm{P}$, Knudsen $\mathrm{P}$, et al: Antioxidant treatment with $N$-acetylcysteine during adult respiratory distress syndrome: A prospective, randomized, placebo-controlled study. Crit Care Med 1992; 20:918-923

14. Bernard GR: $N$-Acetylcysteine in experimental and clinical acute lung injury. Am J Med 1991; 91:54-59

15. Schmidt W, Walther A, Gebhard MM, et al: Influence of $N$-acetylcysteine treatment on endotoxin-induced microcirculatory disturbances. Int Care Med 1998; 24:967-972

16. Faist E, Schinkel C, Zimmer S: Update on the mechanisms of immune suppression of injury and immune modulation. World J Surg: 1996; 20:454-459

17. Klebanoff SJ: Oxygen metabolism and the toxic properties of phagocytes. Ann Intern Med 1980; 93:480-487

18. Koch T, Heller S, Heissler S, et al: Effects of $N$-acetylcysteine on bacterial clearance. Eur $J$ Clin Invest 1996; 26:884-92

19. Koch T: Antioxidative status in patients with acute respiratory distress syndrome. Intensive Care Med 1999; 25:134-136

20. Members of the American College of Chest Physicians/Society of Critical Care Medicine, Consensus Conference Committee: Definitions of sepsis and organ failure and guidelines for the use of innovative therapies in sepsis. Crit Care Med 1992; 20:864-874

21. Emmendörfer A, Hecht M, Lohmann- Matthes M-L: A fast and easy method to determine the production of reactive intermedi- ates by human and murine phagocytes using dihydrorhodamine 123. J Immunol Methods 1990; 131:269-75

22. Rothe G, Kellermann W, Valet G: Flow cytometric parameters of neutrophil function as early indicators of sepsis- or trauma related pulmonary or cardiovascular organ failure. J Lab Clin Med 1990; 115:52-61

23. Knaus WA, Draper EA, Wagner DP, et al: APACHE II: A severity of disease classification system. Crit Care Med 1985; 13: 818-829

24. Wheeler AP, Bernard GR: Current concepts: Treating patients with severe sepsis. $N$ Engl J Med 1999; 340:207-214

25. Penn RL, Maca RD, Berg RD: Increased translocation of bacteria from the gastrointestinal tract of tumor bearing mice. Infect Immun 1985; 47:793-798

26. Borgstöm L, Kagedal B, Paulsen O: Pharmacokinetics of $N$-acetylcysteine in man. Eur J Clin Pharmacol 1986; 31:217-222

27. Urban T, Akerlund B, Jarstrand C, et al: Neutrophil function and glutathione-peroxidase (GSH-px) activity in healthy individuals after treatment with $N$-acetyl-L-cysteine. Biomed Pharmacother 1997; 51:388-390

28. Andersen LW, Thiis J, Rygg I: The role of $\mathrm{N}$-acetylcysteine administration on the oxidative response of neutrophils during cardiopulmonary bypass. Perfusion 1995; 10:21-26

29. Kharazmi A, Nielsen H, Schiotz PO: $N$ Acetylcysteine inhibits human neutrophil and monocyte chemotaxis and oxidative metabolism. Int J Immunopharmacol 1988; 10: $39-46$

30. Verhasselt V, Vanden Berghe W, Vanderheyde $\mathrm{N}$, et al: $N$-Acetyl-L-cysteine inhibits primary human $\mathrm{T}$ cell responses at the dentritic cell level: association with NF-kappaB inhibition. J Immunol 1999; 162:2569-2574

31. Khachigian LM, Collins T, Fries JW: $N$-Acetyl cysteine blocks mesangial VCAM-1 and NFkappaB expression in vivo. Am J Pathol 1997; 151:1225-1229

32. Faruqi RM, Poptic EJ, Faruqi TR, et al: Distinct mechanisms for $N$-acetylcysteine inhibition of cytokine-induced E-selectin and VCAM-1 expression. Am J Physiol 1997; 273: H817-H826

33. Linden M, Wieslander E, Eklund A, et al: Effects of oral $\mathrm{N}$-acetylcysteine on cell content and macrophage function in bronchoalveolar lavage from healthy smokers. Eur Respir J 1988; 1:645-650

34. Ohmann L, Dahlgren C, Follin P, et al: $N$ Acetylcysteine enhances receptor mediated phagocytosis by human neutrophils. Agents Actions 1992; 36:271-277

35. Duignan JP, Collins PB, Johnson AH, et al: The association of impaired neutrophil chemotaxis with post-operative surgical sepsis. Br J Surg 1986; 73:238-240

36. Buffone V, Meakins JL: Christou NV: Neutrophil function in surgical patients: Relationship to adequate bacterial defenses. Arch Surg 1984; 119:39-43

37. Kinnaert P, Van Geertruyden N, DePauw L, et al: Modulation of inflammatory reactions by surgical trauma: lack of relationship with corticosteroid secretion. World J Surg 1989; 13:478-483

38. Wakefield CH, Carey PD, Fould S, et al: Polymorphonuclear leukocyte activation an early marker of the postsurgical sepsis response. Arch Surg 1993; 128:390-395

39. Davies M, Sheppard K, Fletcher J: The effects of surgery on the activity of neutrophil granule proteins. Br J Haematol 1983; 53:5-13

40. Utoh J, Yamamoto T, Utsunomiya T, et al: Effect of surgery on neutrophil functions, superoxide and leukotriene production. $\mathrm{Br} J$ Surg 1988; 75:682-685 